\title{
Photon-axion conversion in low symmetrical dielectric structures
}

\section{Gorelik V.S.}

P. N. Lebedev Physical Institute of RAS, Moscow, Russian Federation;

E-mail: Gorelik<gorelik@sci.lebedev.ru>;

The opportunities for detecting of dark matter elementary particles - axions - are discussed. In accordance with the predictions of the modern theory, these particles have a very small rest mass, corresponding to the energy range $0.001 \ldots 1.0 \mathrm{meV}$. At the presence of magnetic field in vacuum the possibility of laser radiation conversion into axions and reverse processes as a result of Primakov effect ("Light shining through wall") is predicted by modern theory. It is proposed to implement photon-axion conversion by using of powerfull pulsed laser as the pumping source. It is proposed to use Raman-active media for photon-axion conversion observation. In the case of Stimulated Raman Scattering the effective photon-axion conversion is predicted. The conditions of synchronism in elementary processes of photon-axion conversion may be realized for so called unitary polaritons, for which the refractive index is close to unity,

Keywords: axion, paraphoton, resonator, conversion, Raman scattering, rest mass, generation, wall.

DOI: $10.18698 / 2309-7604-2015-1-171-186$

\section{Introduction}

According to the modern concepts of high energy physics [1-4] about the scenarios of evolution of the Universe, after the initial homogeneous and isotropic state physical vacuum has undergone phase transition, resulting in the decreased symmetry vacuum. The so-called standard model of this phase transition based on the use of local (gauge) symmetry, asked the group SU2 $\times$ $\mathrm{U}(1)$. The conclusion of this theory is prediction of formation in vacuum scalar field that specifies the symmetry of the low-temperature phase and leading to the formation of massive elementary particles. In particular, in the spectrum at high energies it is predicted the presence of scalar particle - the Higgs boson [4-7], the search for which in recent years intensively carried out on the experimental setup, generating elementary particles with energy exceeding $1 \mathrm{TeV}$. Such a particle is expected to be found in the result of the analysis allowed by the selection rules of the processes of decay of a scalar boson into pairs of gamma-quanta, the presence of which may be installed by conventional methods known in high energy physics. Along with scalar Higgs boson, also called as amplitudon, the theory of phase transition in vacuum predicts massless boson (phason), called as Nambu - Goldstone boson. In recent years it became clear that the standard model, despite for great success in the description of the spectrum of the known elementary particles spectrum, requires further clarification, by taking into account the effects of time (T) breaking symmetry at small distances. In this regard, the theory, predicts that the rest mass of 
phason must be different from zero. So the conclusion was done about the existence of elementary particles with the pre-separately small but finite rest mass [8-11]. One example of such particles can be axions [1]. It is expected that the rest mass axion corresponds to the energy range of $0.001 \ldots 1.0 \mathrm{meV}$, i.e. should being substantially less than the rest mass of all known elementary particles. The axions are pseudoscalar particles, i.e. their wave function changes sign under inversion and mirror reflections space. The actual topics of modern physics is also associated with prediction of presence in the Universe dark matter (total share 0,23) and dark energy (the total share of 0.73). Recently on the basis of astrophysical data hypothesized [12-17] that candidates for the role of particles dark matter are axions - pseudoscalar particles with preseparately low rest mass and relativistic dispersion law. "Cold" (slow) axions are nonrelativistic (Newtonian) particles, being at a state of Bose-Einstein condensate. "Hot" (fast) axions are relativistic particles and move with a speed close to the speed of light. Important property of these particles - their superweak interaction with material environments similar to the neutrinos. According to the estimates obtained from astrophysical data, the equilibrium concentration of axions in our part of the galaxy is about $10^{-24} \mathrm{~g} / \mathrm{cm} 3$. With this concentration owing to the extremely small rest mass axions must be in Bose-Einstein condensate state even at room temperature.

Some scientists proposed the detection and generation for axions in the laboratory conditions [12-17]. In this the direction analyzed the feasibility of photon - axion conversion and inverse processes allowed by the selection rules in the presence of strong external magnetic field. In this case explores two effects: 1) the generation of "hot" axions in the con-version of laser photons or x-ray radiation into axions of the same energy; 2) the detection of "cold" (Newtonian) axion when their conversion into photons of the microwave range. To now received only the first experimental results in these area, requiring optimization of the conditions of observation and finding ways to improve the efficiency of the discussed processes for reliable interpretation of the obtained experimental results.

This paper presents new experimental scheme to implement the generation and detection for axions and analyzed the possibilities of their implementation in the laboratory.

\section{To the theory of photon-axion conversion in vacuum}

In [18-21] on the dynamics of stars with masses $8 . .12$ Solar masses, the conclusion was made about the processes of photon-axion conversion properties inside stars and estimates the efficiency of photon-axion conversion elemental process. Furthermore, in [10-12, 15] it was suggested the possibility of "hot" axions creating with energy $2-3 \mathrm{eV}$ in the laboratory when 
implementing experiments of the type "Light shining through wall" (so-called Primakov-effect). Schematic diagram for the generation and detection for axions using the Primakov effect is shown in Fig. 1.

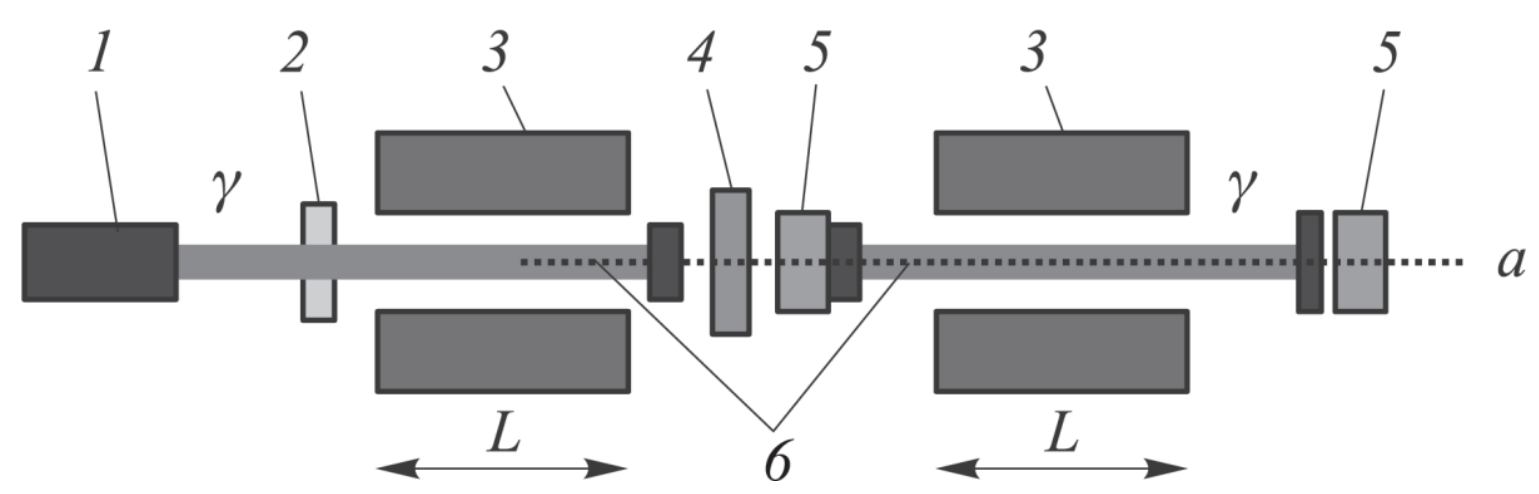

Fig. 1. Schematic setup for observation of the effect of

photons of laser radiation conversion into pseudoscalar bosons $\gamma \rightarrow$ a (axions) and the reverse process — the reconversion a $\rightarrow \gamma ; 1$ - the source of laser radiation; 2 - translucent mirror; 3

— solenoids; 4 - opaque wall; 5 - receivers of the secondary radiation; 6 -

Fabry - Pero resonator, generating the light quanta $(\gamma)$.

Thus at the first step with the help of modern lasers of visible range, generation of photons $(\gamma)$ takes place, taking into account the strong magnetic field presence (see Fig.1), the photonaxion $(\gamma$-a) conversion should take place . Axions, emerging at left side of setup, should penetrate through the opaque wall 4. Then, at right part of setup, reverse processes should take place resulting axion-photon conversion. So the reconverted photons may be detected after opaque wall. As a result of the secondary photons detection the probability of these processes and effectiveness of the photon production from "hot" for axions in the laboratory may be evaluated. According to the selection rules, photon-axion conversion processes are permitted only when constant magnetic field, the induction of which is perpendicular to the direction of the beam of exciting radiation, is applied to the area of laser radiation. In the absence of an external magnetic field only three-particle conversion processes are allowed, the probability of which is very small. As it turned out from the experiments $[10-12,15]$, the useful signal of the secondary radiation produced as the result of conversion-reconversion processes seemed to be extremely small and that during the experiments is below the sensitivity threshold of the modern detectors. The photon-axion coupling constant $\mathrm{g}$, bounded with the probability of conversion- reconversion processes, is very small $[10-12,15]: \mathrm{g}$ $\approx 10-10 \mathrm{GeV}-1$. 
As analogous to the processes of photon-axion conversion we consider corresponding Raman scattering (RS) of light processes in crystals or dielectric media [10-12, 15, 21-23]. Fig. 2a,b illustrates the Stokes (a)and anti-Stokes processes in condensed dielectric media and corresponding photon-axion conversion (c) and reconversion (d) processes.

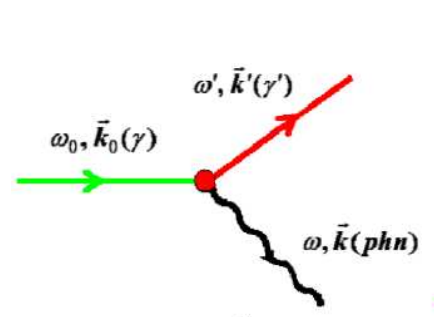

$a$
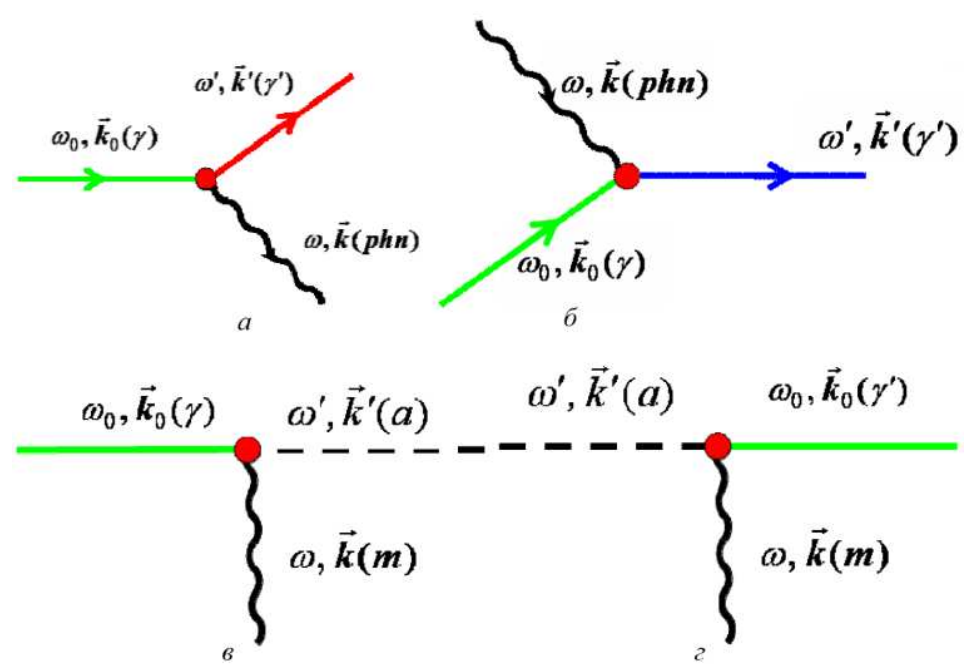

Fig. 2. Schemes of elemental Stokes(a) and anti-Stokes Raman (b) in condensed media and also corresponding photon-axion conversion (c) and reconversion (d) processes.

For the Stokes and anti-Stokes Raman elemental processes momentum and energy conservation laws may be presented as:

$$
\begin{aligned}
& \hbar \omega_{0}=\hbar \omega^{\prime}+\hbar \omega ; \\
& \hbar \vec{k}_{0}=\hbar \vec{k}^{\prime}+\hbar \vec{k} ; \\
& \hbar \omega_{0}+\hbar \omega=\hbar \omega^{\prime \prime} ; \\
& \hbar \vec{k}_{0}+\hbar \vec{k}=\hbar \vec{k}^{\prime \prime} .
\end{aligned}
$$

Here $\hbar \omega_{0}, \hbar \omega^{\prime}, \hbar \omega^{\prime \prime}, \hbar \omega$ - the energies of exciting photon of laser emission, photon of Stokes emission, photon of anti-Stokes emission and phonons correspondingly; $\hbar \vec{k}_{0}, \hbar \vec{k}^{\prime}, \hbar \vec{k}^{\prime \prime}, \hbar \vec{k}$ corresponding quasimomentums. For Stokes and anti-Stokes conversion-reconversion processes accordingly we have: 


$$
\begin{aligned}
& \hbar \omega_{0}(\gamma)=\hbar \omega^{\prime}(a)+\hbar \omega(m) ; \\
& \hbar \vec{k}_{0}(\gamma)=\hbar \vec{k}^{\prime}(a)+\hbar \vec{k}(m) ; \\
& \hbar \omega^{\prime}(a)+\hbar \omega(m)=\hbar \omega_{0}(\gamma) ; \\
& \vec{k}^{\prime}(a)+\hbar \vec{k}(m)=\hbar \vec{k}_{0}(\gamma) .
\end{aligned}
$$

Here $\hbar \omega_{0}(\gamma), \hbar \omega^{\prime}(a), \hbar \omega(m)$ - the energies of exciting photon of laser emission, axion and quantum of indirect magnetic field emission correspondingly; $\hbar \vec{k}_{0}(\gamma), \hbar \vec{k}^{\prime}(a), \hbar \vec{k}(m)$ corresponding quasimomentums. When the conversion of photons into axions in the constant external magnetic field takes place, instead of (2) relations, we have:

$$
\begin{aligned}
& \hbar \omega_{0}(\gamma)=\hbar \omega^{\prime}(a) \\
& \hbar \vec{k}_{0}(\gamma)=\hbar \vec{k}(a) \\
& \hbar \omega^{\prime}(a)=\hbar \omega_{0}(\gamma) \\
& \hbar \vec{k}^{\prime}(a)=\hbar \vec{k}_{0}(\gamma)
\end{aligned}
$$

Thus, photon-axion conversion and reconversion processes in magnetic field are similar to the processes of Stokes and anti-Stokes Raman processes in condensed media. The full probability(1/s) of Stokes RS may be presented as [22]:

$$
W_{n_{s}+1, n+1}^{(s)}=W_{s p}\left(n_{s}+1\right)(n+1)
$$

Here $n_{s}, n$-the quantum figures of Stokes photons and phonons. Thus for Stimulated Raman Scattering (STRS) intensity it takes place:

$$
I^{\text {STRS }}=I^{\text {SPRS }} n_{s}
$$

where $I^{S P R S}$ - the intensity of the corresponding spontaneous RS. At sufficiently high exciting laser intensity, STRS may be observed and in this case the intensity of STRS may be presented as:

$$
I^{\text {STRS }}=I^{\text {SPRS }} \exp \left(\alpha I_{0} I\right)
$$


where $I_{0}$ - the intensity of exciting laser emission, $l$-the length of working medium, $\alpha$-increment of STRS. The typical value of $\alpha$ is: $\sim 0,01 \mathrm{~cm} / \mathrm{Mw}$. So for $l=1 \mathrm{~cm}$ and $I_{0}=10^{8} \mathrm{~W} / \mathrm{cm}^{2}$ the intensity of STRS is: $I^{\text {STRS }}=0.01 I_{0}$. Under excitation of RS by ultra short $\left(10^{-10} \mathrm{~s}\right)$ laser pulses $(\lambda=0.532 \mu)$ at peak power $I_{0}=10^{12} \mathrm{~W} / \mathrm{cm}^{2}$, we have $n_{s} \approx 10^{14}$.

Lagrangian density of the electromagnetic field at the presence of magnetic field may be presented as [11-17]:

$$
\mathfrak{I}=-\frac{1}{4} F_{\mu \nu} F^{\mu v}+\frac{1}{2}\left(\partial_{\mu} \phi_{a} \partial_{\mu} \phi_{a}-m_{a}^{2} \phi_{a}^{2}\right)-\frac{1}{2} g \phi_{a} F_{\mu \nu} \tilde{F}^{\mu v}
$$

Here $F_{\mu \nu}, F^{\mu \nu}$-tensors of electromagnetic field, $\tilde{F}^{\mu \nu}=\frac{1}{2} \varepsilon_{\mu \nu \lambda \rho} F^{\lambda \rho}, \phi_{a}$-wave function of axion, $g-$ the interaction fields constant. Will continue to use the system of units for which the fundamental constants $\mathrm{C}_{0}$ (velocity of light in vacuum) and $\hbar$ are equal to unity. Based on (8), the equations of motion for the respective fields we write in the form:

$$
\partial_{\mu} F_{\mu v}=g \partial_{\mu}\left(\phi_{a} \tilde{F}^{\mu v}\right) ;\left(\partial_{\mu} \partial^{\mu}+m_{a}\right) \phi_{a}=g B_{0} E
$$

The solution (8) for pseudoscalar fields $\phi_{a}$ has the form:

$$
\phi_{a}^{ \pm}(r, t)=e^{-i \omega t} \int d^{3} r^{\prime} \frac{1}{4 \pi} \frac{\exp \left( \pm i \overrightarrow{k_{a}}\left(\vec{r}-\vec{r}^{\prime}\right)\right.}{\left|\vec{r}-\vec{r}^{\prime}\right|} g \vec{B}_{0} \vec{E}
$$

In the one-dimensional case, the solution of (10) can be written as follows:

$$
\phi_{a}^{+}(r, t)=i E_{0}\left(g B_{0} l / 2 k_{a}\right) F(q) e^{i\left(k_{a} x-\omega t\right)} .
$$


Here $q=\left(\omega-k_{a}\right)$ - momentum, transmitted to magnetic field; $F(q)=\frac{\sin q l / 2}{q l / 2}$, i.e. $F(0)=1$.

The likelihood of occurrence $\mathrm{Na}$ pseudoscalar bosons in the result of its conversion from $\mathrm{N} \gamma$ quanta (photons) of exciting radiation is given by the equation

$$
P_{\gamma \rightarrow a}=\frac{N_{a}}{N_{\gamma}}=\frac{1}{4}\left(\frac{\omega}{k_{a}}\right)\left(g B_{0} I\right)^{2} F^{2}(q) .
$$

If we used the following value of parameters [4-7]: $g \sim 10^{-10} \mathrm{GeV}^{-1}, \mathrm{~B}=10 \mathrm{Tl}, l=1 \mathrm{~m}$, it takes place:

$$
P_{\gamma \rightarrow a} \sim 10^{-18}
$$

When using of Fabry - Perot resonator (see Fig. 1) with finesse $\mathrm{Q}=10^{4}$, conversion probability enhances to value:

$$
P_{\gamma \rightarrow a}=\frac{1}{4}\left(\frac{Q}{\pi}\right)\left(\frac{\omega}{k_{a}}\right)\left(g B_{0} I\right)^{2} F^{2}(q) \sim 10^{-14}
$$

In this case the full probability of reconversion is:

$$
P_{\gamma \rightarrow \gamma^{\prime}}=\left(P_{\gamma \rightarrow a}\right)\left(P_{a \rightarrow \gamma^{\prime}}\right) \sim 10^{-28}
$$

When the power of the exciting radiation of the argon laser is about $10 \mathrm{~W}$, operating in continuous or quasi-continuous manner $(\lambda=0.514 \mu \mathrm{m})$, the number of excitatory quanta of light entering in the first cavity during one second is $\mathrm{N} \gamma \approx 10^{20} 1 / \mathrm{s}$. Accordingly, the output (see Fig. 1) according to relation (11), i.e. the number of photons after reconversion process is $\mathrm{N} \gamma^{\prime} \approx 10^{-}$ ${ }^{8} 1 / \mathrm{s}$. This corresponds to a noise level and is below the limit of sensitivity light radiation detectors. Thus, observation of the discussed effect in the regime of spontaneous processes is not promising. 


\section{Stimulated photon-axion conversion processes}

For stimulated conversion process by analogy with the STRS (relations (6), (7)), we obtain:

$$
P_{\gamma \rightarrow a}=\frac{1}{4} n_{a}\left(\frac{Q}{4 \pi}\right)\left(\frac{\omega}{k_{a}}\right)\left(g B_{0} I\right)^{2} F^{2}(q) .
$$

For $\mathrm{n}_{\mathrm{a}}$ value we use the relation:

$$
n_{a}=\frac{1}{4}\left(\frac{Q}{\pi}\right)\left(\frac{\omega}{k_{a}}\right)\left(g B_{0} I\right)^{2} F^{2}(a) n_{\gamma}
$$

Here $-n_{\gamma}$ quantum figure of exciting radiation. For the excitation of stimulated conversion processes is necessary to use the short $\left(10^{-8} \mathrm{~s}\right)$ or ultrashort $\left(10^{-10} \mathrm{~s}\right)$ pulses of exciting radiation [21-23]. Consider a case where the power density of the exciting pulse is $10^{12} \mathrm{~W} / \mathrm{cm}^{2}$, and its duration is $10^{-10} \mathrm{~s}$. This type of operation can be implemented for the second optical harmonic solid-state YAG: $\mathrm{Nd}^{3+}$ laser. The number of quanta per the fashion of the exciting radiation field is $\mathrm{n}_{0} \approx 10^{14}$. According (15), we obtain $\mathrm{n}_{\mathrm{a}} \approx 1$, i.e., in such conditions it is possible to expect impletion of threshold regime of stimulated conversion processes. In this case the total probability of conversion-reconversion is:

$$
P_{\gamma \rightarrow a}=\frac{1}{4} n_{a}\left(\frac{Q}{4 \pi}\right)\left(\frac{\omega}{k_{a}}\right)\left(g B_{0} I\right)^{2} F^{2}(\mathrm{q})
$$

Under the condition of transition from spontaneous emission to stimulated one the number

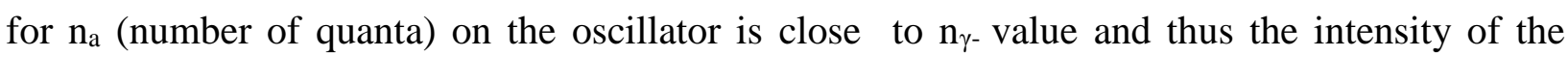
secondary emission should be large enough for detection.

\section{Proposed experimental scheme for "hot" axions detection in media}

Another possibility to improve the efficiency of photon-axion and axion-photon conversion processes is using the dielectric media, inserted in magnetic field. In this case there is a problem of compliance with the conditions of synchronism (2)-(4). This problem is solved by tuning the frequency of the photons involved in the process of photon-axion conversion to the frequencies of 
the so-called unitary polaritons, for which module the refractive index is close to unity. Very promising material for this task are the ruby crystals — the active medium for laser generation. Based on three resonance transitions in ions of chromium the dispersion of polaritons (see Fig.3) in this the crystal can be represented as:

$$
\omega^{2}=\frac{c_{0}^{2} k^{2}}{\varepsilon(\omega)} ; \varepsilon(\omega)=\varepsilon_{\infty} \prod_{j=1}^{j=3} \frac{\left(\omega_{l j}^{2}-\omega^{2}\right)}{\left(\omega_{t j}^{2}-\omega^{2}\right)}
$$

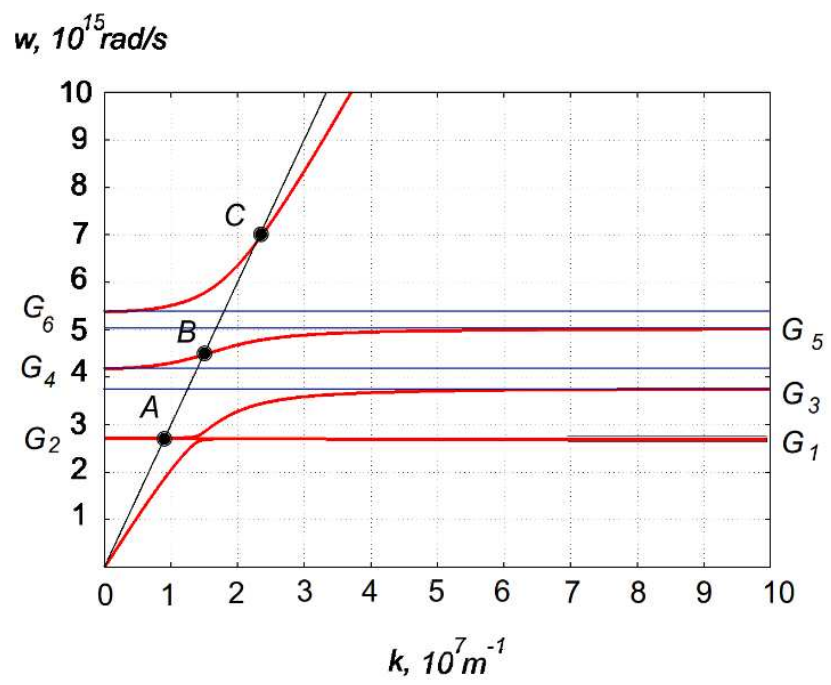

Fig.3. Polariton dispersion law in ruby crystal; $\mathrm{G}_{1}, \mathrm{G}_{3}, \mathrm{G}_{5}$ points correspond to transversal (t) excitations with frequencies $\omega_{t j}, \mathrm{G}_{2}, \mathrm{G}_{4}, \mathrm{G}_{6}$ points - to longitudinal (l) excitations with frequencies $\omega_{l j}$,

We can see from Fig.3, that group velocity at point A is very small. Ruby laser generation, corresponding to $2 \mathrm{E} \rightarrow 4 \mathrm{~A}$ transition of $\mathrm{Cr}^{3+-}$ ion, has wavelength $\lambda=694,3 \mathrm{~nm}$ (point $\mathrm{G}_{1}$ ). Thus, the active medium of the ruby laser, generating radiation with wavelength $\lambda=694,3 \mathrm{~nm}$ can be directly used for the process of photon-axion if magnetic field is applied. Fig. 4a-c illustrate the corresponding experimental schemes for photon-axion conversion processes realizing with ruby laser radiation using. 


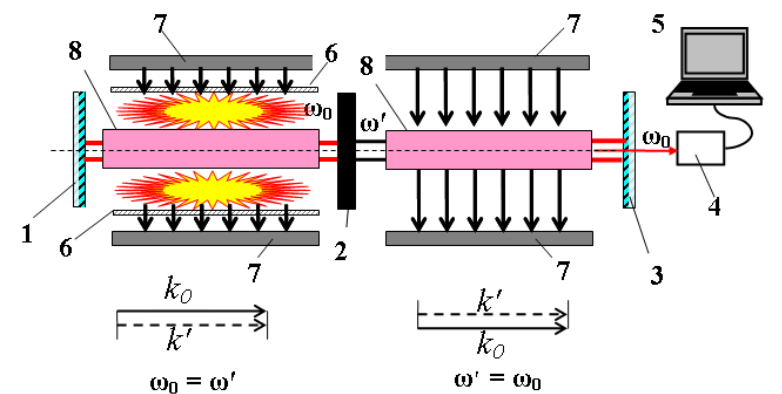

a

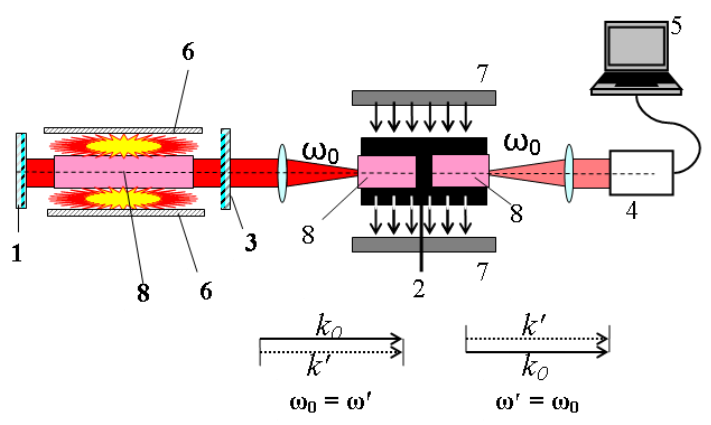

$\mathrm{b}$

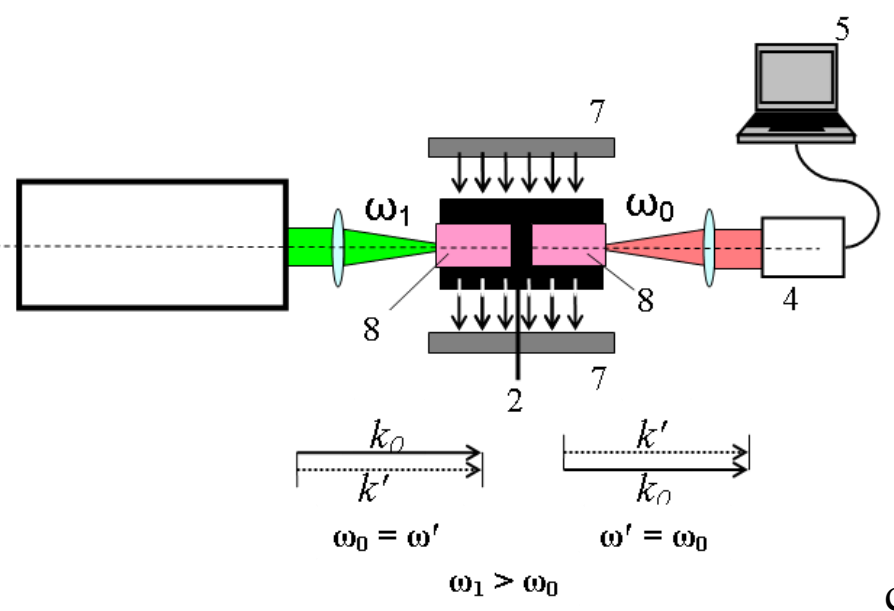

Fig. 4. Proposed experimental schemes (a-c) for photon-axion conversion observation with using of ruby crystal; 1,3- mirrors, 2-opaque wall, 4-detector,5-computer, 6-reflector,7-magnet,8- ruby crystal.

At Fig.4a opaque wall is used as the second mirror in laser resonator; Fig.4b corresponds to scheme, two ruby crystal samples $\left(\mathrm{Al}_{2} \mathrm{O}_{3}: \mathrm{Cr}^{3+}\right)$ are used outside of laser resonator; Fig.4c illustrate the scheme, additional laser source (violet or green) for luminescence excitation is used.

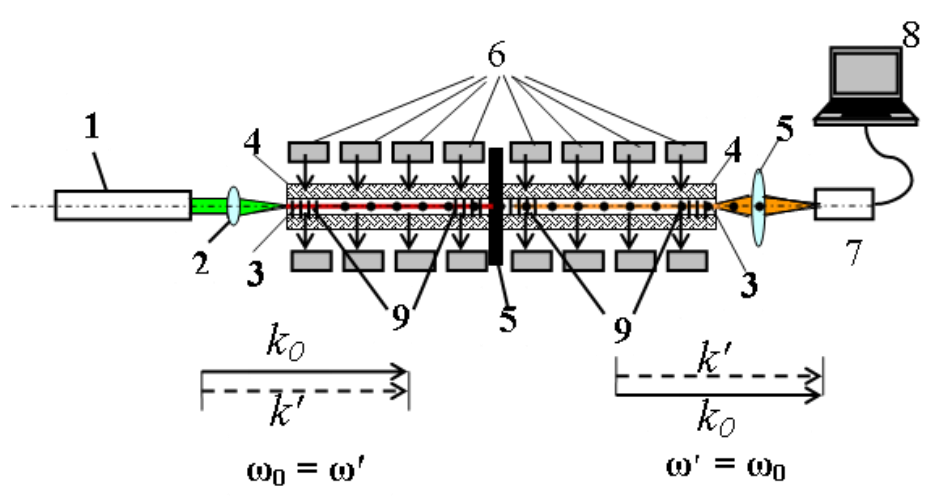


Fig. 5. Proposed experimental schemes for photon-axion conversion observation with using of straightforward quartz fiber $\mathrm{SiO}_{2}: \mathrm{Er}^{3+}$; 1-laser, 2-lens, 3- quartz fiber $\mathrm{SiO}_{2}: \mathrm{Er}^{3+}$; 4-holder, 5opaque wall, 6- magnet, 7--detector,8-computer.

The probability of observation of processes photon-axion and axion-photon conversion at experimental schemes, presented at Fig. 4a-c, is expected higher than in vacuum (see Fig. 1),since the unitary polaritons (point A at Fig.4) group velocity is considerably less than the speed of light in vacuum. Another opportunity for enhancing of photon-axion conversion efficiency gives the fiber laser equipment (see Fig.5). In this case exciting laser emission falls into straightforward quartz fiber $\mathrm{SiO}_{2}: \mathrm{Er}^{3+}$, placed inside of magnetic field. Due to very high density of laser light inside of fiber and long enough its size the sensitivity of such set up is waited high enough.

An additional opportunity to improve the efficiency photon-axion conversion can be realized with utilizing of photonic crystal [21-29]. In such crystals unitary photons (polaritons) are present in the infrared, visible and ultraviolet regions.areas. Group velocity of unitary polaritones (point $U$ at Fig.6)) can be anomalously low that causes a change in the probability of spontaneous emission processes [30] and lower thresholds of the relevant processes [31-39].

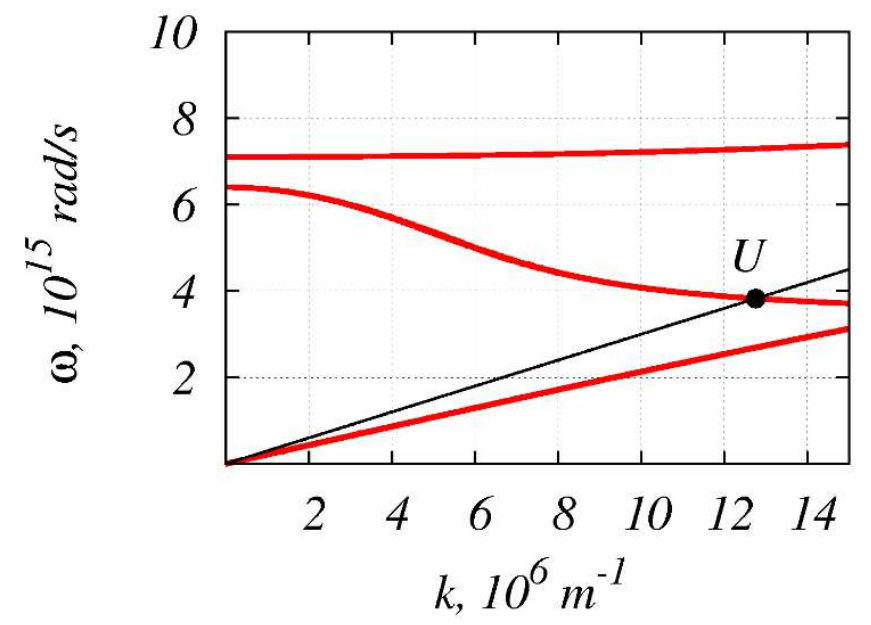

Fig.6. Schematic shape of electromagnetic waves dispersion law in photonic crystal. Point U corresponds to unitary polaritons. 


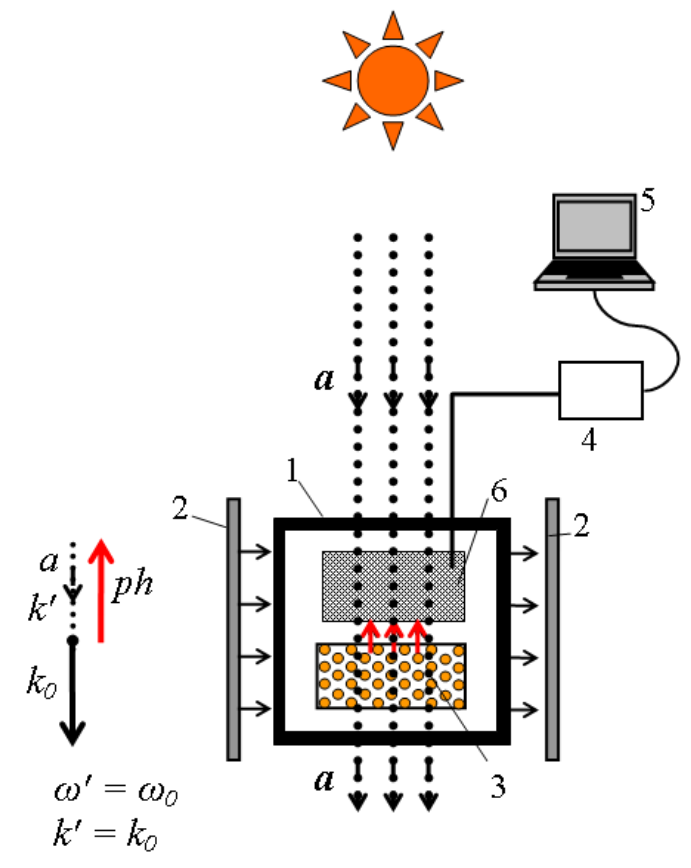

Fig.7. Proposed experimental setup for detection of sun hot axions; 1-box, 2-magnet,3-photonic crystal,4-amplifier,5-computer, 6-detector.

Fig.7 illustrates the proposed experimental scheme for detection of sun hot axions, with using of photonic crystal, inserted into closed box and magnetic field.

At last, note that the likelihood of photon-axion conversion may be essentially enhanced in Raman active media, at the conditions of Stimulated Raman Scattering or in the case of Resonance Raman scattering. In these cases, very strong photon-phonon interaction takes place resulting in two-photon bounding in media and axion generation in vacuum.

\section{The detection of "cold" axions}

The task of slow (cold) axions detection is connected with the microwave emission registration, energy of which quanta $((0.001-1 \mathrm{meV}))$ is coincided with cold axions energy. At the presence of strong enough magnetic field (1-10 Tl) microwave photons should appear in closed box as a result of axion-photon conversion processes. As the receiver of microwave photons may be used superconductor detector at scheme, illustrated by Fig.8. 


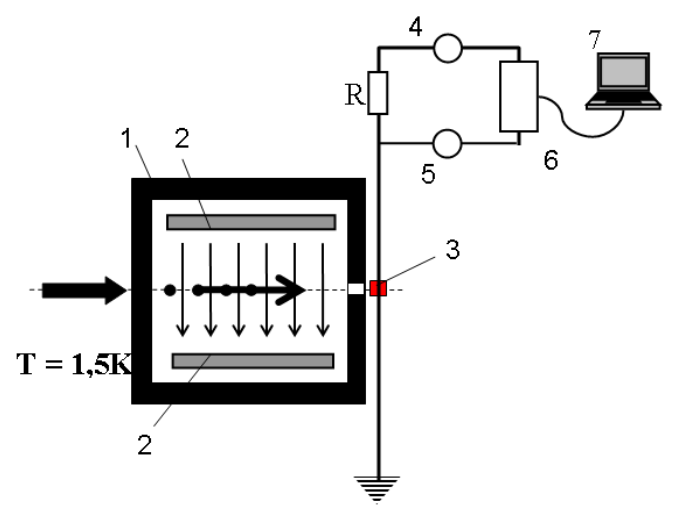

Fig.8. Experimental setup for superconductor detection of microwave photons, appearing in closed box, inserted in magnetic field at low temperature.

Recently the opportunity of detection of cold axion on the base of Josephson effect was analyzed [40 - 45]. The registration of so cold Shapiro steps permitted to evaluate [45] the rest mass $(0.11 \mathrm{meV})$ of axions and its density around us $\left(0.05 \mathrm{GeV} / \mathrm{cm}^{3}\right)$.

\section{Summary}

Thus it is proposed the optimization of experimental installations for the detection of "hot" and "cold" axions, presumably a dark elementary particles matter. For the generation of "hot" axions it is proposed to use pulsed laser light sources characterized by high spectral intensity in the visible or ultraviolet ranges. This ensures that the transition from the regime spontaneous photon-axion conversion processes to stimulated one, similar to processes of stimulated Raman scattering of light. The rest mass of axions corresponds to the range $0.001 \ldots 1.0 \mathrm{meV}$. It was shown that photon-axion conversion efficiency may be enhanced if some dielectric media use as ruby crystal or fiber, saturated by rare earth elements. New opportunities, presented by photonic crystals, permit the essential enhance of the spontaneous and stimulated process efficiency. The detectors of microwave photons, appearing as the result of the conversion of "cold" axion into microwave photon, are proposed on the base superconductor plate or Josephson bridge.

The work was supported by the Russian Foundation for Basic Research (projects 13-02-00449, 14-02-00190, 14-02-90406, 14-02-90007) and by the Belarusian Republican Foundation for Fundamental Research (project no. F14R_091). 


\section{References}

1. Ryabov V.A., Tsarev V.A., Tskhovrebov A.M. (2008). The search for dark matter particles.

Uspehi fizicheskih nauk [Achievements of physical sciences], vol. 51, no. 11, 1091-1121.

2. Appelquisi T., Cheng H-C. Dobrescu. B.A (2001). Phys.Rev., D 62, 035002.

3. Servant G., Tait T.M. (2001). Nucl. Phys., B 650, 391.

4. Goldstone J., Salam A., Weinberg S. (1962). Phys. Rev., 127, 965.

5. Kim J.E., Garosi G. (2010). Reviews of modern physics, Vol. 82, 557-601.

6. Hoffmann S. (1986). Phys. Lett., B 193, 117- 122.

7. Picciotto C., Pospelov M. (2005). Phys. Let., B 605, 15.

8. Okun L. B. (1982). Zhurnal jeksperimental'noj i teoreticheskoj fiziki [Journal of experimental and theoretical physics], 83, 892-898.

9. Jaeckel J., Redondo J., Ringwald A. (2009). EPL, 87, 10010.

10. Sikivie P., Tanner D.B., van Bibber K. (2007). Phys. Rev. Lett., 98, 172002.

11. van Bibber K.A., Dagdeviren N.R., Koonin S.E., Kerman A.K., Nelson H.N. (1978). Phys. Rev. Lett., 59, 759-762.

12. Duffy L. D., Sikivie P., Tanner D. B., Asztalos S. J., Hagmann C., Kinion D., Rosenberg L. J, van Bibber K.,. Yu D. B, Bradley R. F. (2006). Physical Review, D 74, 012006.

13. Rosenberg I.J., Bibber K.A. (2000). Phys. Rep, 325, 1.

14. Stancil D. D. (2007). Physical Review, D 76, 111701(R).

15 Afanasev A., Baker O.K., Beard K.B., Biallas G., Boyce J., Minarni M., Ramdon R., Shinn M., Slocum P. (2008). Phys. Rev. Lett., 101, 120401.

16. Mueller Guido, Sikivie Pierre, Tanner D.B., van Bibber K. (2009). Physical Review, D 80, 072004.

17. Sikivie P., Tanner D.B., Bibber K. (2007). Phys. Rev. Lett. 98, 172002.

18. Hoskins J., Hwang J., Sikivie P., Tanner D. B. (2010). Phys. Rev. Lett. 104, 041301.

19. Friedland A., Giannotti M., Wise M. (2013). Phys. Rev. Lett. 110, 061101.

20. Bellini G., Benziger J., Bick D. (2012). Phys. Rev., D, 85, 092003.

21. Hudson H. S., Acton L. W., DeLuca E., Hannah I. G., Reardon K., Van Bibber K. (2012). $\operatorname{arXiv,~} 1201.4607 \mathrm{v} 1$.

22. Gorelik V.S. (2010). European Journal - Applied Physics, 49(3), 3307.

23. van Bibber K., Dagdeviren N.R., Koonin S.E., Kerman A.K., Nelson H.N. (1987). Phys. Rev. Lett., 59, 759-762. 
24. Duffy L.D., Sikivie P., Tanner D.B., Asztalos S.J., Hagmann C., Kinion D., Rosenberg L.J, van Bibber K., Yu D.B.,. Bradley R.F. (2006). Physical Review, D 74, 012006.

25. Sikivie P., Tanner D.B., van Bibber K. (2007). Phys. Rev. Lett., 98, 172002.

26. Afanasev A., Baker O.K., Beard K.B., Biallas G., Boyce J., Minarni M., Ramdon R., Shinn M., Slocum P. (2008). LIPSS Collaboration. Phys. Rev. Lett., 101, 120401.

27. Gorelik V. S., Sushchinskii, M. M. (1969). Raman scattering of light in crystals. Uspehi fizicheskih nauk [Achievements of physical sciences], 98, 237.

28. Gorelik V. S. (2010). Linear and nonlinear optical phenomena in nanostructured photonic crystals, filled by dielectrics or metals. Eur. Phys. J. Appl. Phys, 49, 330007.

29. Gorelik V. S., Izmailov G.N. (2011). Stimulated Photon Conversion into Pseudo-Scalar Bosons. Bull. Lebedev Phys Inst, 38, No. 6, 177-183.

30. Yablonovitch E. (1987). Phys. Rev. Lett., 58, 2059.

31. John S. (1987). Phys. Rev. Lett. 58, 2486.

32. Dowling J.P., Bowden C.M. (1992). Phys. Rev., A46, 1, 612.

33. John S., Quang T. (1995). Phys. Rev. Lett., 74, 3419.

34. Astratov V.N., Bogomolov V.N., Kaplyanskii A.A, Prokofiev A.V., Samoilovich L.A., Samoilovich S.M., Vlasov Yu.A. (1995). Nuovo Cimento, D17, 1349.

35. Bogomolov V.N., Gaponenko S.V., Kapitonov A.M., Prokofiev A.V., Ponyavina A.N., Silvanovich N.I., Samoilovich S.M. (1996). Appl. Phys. A63, 6, 613.

36. Purcell E.M. (1946). Phys. Rev. 69, 681.

37 Gorelik, V.S. (2007). Quantum Electronics, 37(5), 409.

38. Gorelik V.S. (2008). Laser Physics, 3, (12), 1479,

39. Voshchinskii, Yu.A., Gorelik V.S. (2011). Inorganic Materials, 47(2), 148.

40. Josephson B.D. (1962). Phys. Lett., 1, 251.

41. Larkin S.Yu. (1999). Izmerenie chastoty monokhromaticheskogo SVCh-polya na osnove nestatsionarnogo effekta Dzhozefsona [Frequency measurement of monochromatic microwave field on the basis of the nonstationary Josephson effect]. Kiev: Naukova Dumka Publ.

42. Shapiro A., Janus R., Holly S. (1964). Rev. Mod. Phys. 36, 223-225.

43. Golovashkin A.I., Elenskiy V.G., Likharev K.K (1983). Effekt Dzhozefsona i ego primenenie [Josephson effect and its application]. Moscow: Nauka Publ [Science]. 
Proceedings of International Conference PIRT-2015

44. Hoffman C., Lefloch F., Sanquer M., Pannetier B. (2004). Physical review, B 70, 180503(R) $(1-4)$.

45. Beck C. (2013). Physical review letters, 111, 231801(1-5). 